\title{
VARIACIÓN ANATÓMICA DE LA MADERA DE AVicenNia Germinans en la LaGuna de La Mancha, Veracruz, MÉXICO
}

\author{
Laura Yáñez-Espinosa ${ }^{1,3}$, Guillermo Angeles ${ }^{1}$, Jorge López-Portillo' ${ }^{1}$, Saamantha Barrales ${ }^{2}$ \\ ${ }^{1}$ Instituto de Ecología, A.C. Km. 2.5 carretera antigua a Coatepec 351, \\ Congregación El Haya, Xalapa 91070, Veracruz, México. \\ ${ }^{2}$ Universidad Veracruzana, Circuito Aguirre Beltrán S/N, Zona Universitaria, Xalapa, Veracruz, México. \\ ${ }^{3}$ Autor para la correspondencia. Dirección actual: Instituto de Investigación de Zonas Desérticas, Facultad de Ingeni- \\ ería, Universidad Autónoma de San Luis Potosí, Altair núm. 200, Col. del Llano, 78377 San Luis Potosí, S.L.P., México. \\ Correo-e: Iyaneze@uaslp.mx; aurora06@prodigy.net.mx
}

\begin{abstract}
Resumen: Avicennia germinans es una especie de mangle que presenta marcada tolerancia a la salinidad, pero sólo se habían observado tendencias a aumentar el tamaño de los caracteres del xilema secundario a bajas concentraciones de salinidad. En la Laguna de la Mancha, Veracruz, esta especie se distribuye en un gradiente de salinidad amplio, lo que hace interesante analizar la variación de sus caracteres anatómicos con respecto a la salinidad. Los resultados sugieren que las diferencias entre sitios para los caracteres a) grosor tangencial de pared de vaso, b) frecuencia de vasos y c) diámetro tangencial del lumen de fibra, se pueden atribuir al efecto del ambiente. El incremento de los tres caracteres anatómicos al aumentar la salinidad y el período de inundación implica una compensación entre eficiencia hidráulica y estabilidad mecánica del xilema. Esto sugiere que el calentamiento global puede afectar a $A$. germinans al aumentar la salinidad debido a la elevación del nivel del mar, afectando su eficiencia en la conductividad hidráulica, reduciendo su crecimiento en altura y en el área foliar.
\end{abstract}

Palabras clave: Avicennia germinans, manglar, variación anatómica, xilema secundario.

\begin{abstract}
Avicennia germinans is a mangrove species with a high tolerance to salinity, but only has shown tendencies towards increase size in wood character at low salinity sites. In La Mancha lagoon, Veracruz, A. germinans is distributed in a wide salinity gradient, making interesting to analyze their anatomical character variation with respect to salinity. The results suggest that differences among sites for a) vessel tangential wall thickness, b) vessel frequency, and c) fiber tangential lumen diameter, could be attributed to an environmental effect. Increase in these three anatomical characters associated with high salinity and increased flooding exposure implies a trade-off between hydraulic efficiency and mechanical stability of wood. This suggests that global warming would affect A. germinans when sea level raise increase salinity, affecting hydraulic conductivity, reducing growth in height and leaf area.
\end{abstract}

Keywords: Anatomical variation, Avicennia germinans, mangrove, secondary xylem.

L os mangles son plantas leñosas caracterizadas por su habilidad común para crecer y prosperar a lo largo de litorales protegidos de las mareas y crecen en sedimentos salinos frecuentemente anaerobios, inundados por aguas salobres, perenne o estacionalmente (Lacerda et al. 1993). Las especies de mangle Rhizophora mangle L., Laguncularia racemosa (L.) C.F. Gaertn. y Avicennia germinans (L.) L., pertenecen a grupos taxonómicos diferentes pero presentan muchas características en común, como lo son su capacidad para adaptarse a las condiciones que se presentan en estos ecosistemas: marcada tolerancia a la salinidad, presencia de raíces modificadas para el intercambio de gases en sustra- tos anaeróbicos y propágulos capaces de flotar, cuyo mecanismo es la dispersión a través del agua (Von Prhal et al., 1990). Particularmente, Avicennia germinans puede crecer en suelos cuya salinidad varía entre 0 y $100 \%$, en suelos cubiertos por unos pocos centímetros de agua continuamente estancada o inundados unas pocas veces por año (Jiménez y Lugo, s.f.).

Las interpretaciones ecológicas del xilema y floema secundarios se basan en correlaciones entre los caracteres anatómicos y los factores ambientales (Baas, 1982) y se han establecido tendencias bien definidas para especies, géneros, familias y floras regionales (Baas, 1986; Carlquist, 
1988; Zhang et al., 1992). Particularmente, la estructura del sistema vascular del tallo tiene una fuerte influencia en la estructura y funcionamiento de las hojas, la ganancia de carbono, la eficiencia en el uso de nutrientes y la tasa de crecimiento (Zahng y Cao, 2009). El estudio de la anatomía de la madera de las especies de mangle resulta atractivo desde el punto de vista ecológico, debido al ambiente tan particular en que habitan, inundado frecuentemente y con alto contenido de sal (Tomlinson, 1986). Un aspecto de gran importancia es el relacionado con las adaptaciones anatómicas de las especies de mangle y la posibilidad de que varíen con las condiciones ambientales (López Portillo y Ezcurra, 2002), ya que identificar la variación anatómica permitirá entender los posibles efectos de la elevación en el nivel del mar como consecuencia del calentamiento global, ya que todas las especies de mangles crecen a lo largo de litorales y evidentemente serán afectados por cualquier cambio en el nivel del mar (Field, 1995).

Robert et al. (2009) estudiaron la anatomía del xilema secundario de Avicennia marina y Rhizophora mucronata en un manglar de la Bahía de Gazi, en Kenia (África occidental), con el fin de encontrar una explicación a la distribución que presentan estas especies en ese manglar. Encontraron que la misma especie presenta diferencias significativas en la frecuencia de vasos, y que esta diferencia está directamente relacionada con la salinidad del sitio.

En Laguncularia racemosa se ha observado variación en la estructura del xilema secundario, principalmente mayor frecuencia de vasos, relacionado con la mayor salinidad y nivel de inundación (Yáñez-Espinosa et al., 2004), pero en A. germinans no se ha observado variación significativa en algunos caracteres de la estructura del xilema secundario del tallo creciendo en un gradiente de salinidad de 6.5 a $10 \%$ y cuatro a ocho meses de inundación (Yáñez-Espinosa et al., 2001).

No obstante, existen evidencias de la variación en caracteres morfológicos y fisiológicos de los árboles de Avicennia germinans. Diversos estudios muestran que los árboles que crecen en áreas con salinidad elevada se caracterizan por el menor tamaño de las hojas, menor altura de la planta, bifurcación más temprana y profusa y la asignación de una proporción más alta de biomasa en las raíces (Soto y Corrales, 1987; Soto, 1988; Suárez y Medina, 2005). Estos cambios morfológicos que se observan en los mangles, causados tanto por la inundación como por la salinidad (entre los que destaca la reducción del área foliar) tienen un impacto en la fisiología de la planta, al disminuir la capacidad fotosintética y la transpiración. Chen et al. (2005) encontraron una disminución del 30 al $40 \%$ en la tasa fotosintética de Lepidium latifolium después de 50 días de inundación, comparada con plantas no inundadas de la misma especie. Esta reducción de la actividad fotosintética debe influenciar el desarrollo del xilema secundario. Asimismo, la inundación prolongada del suelo causa grandes alteraciones en las propiedades fisicoquímicas y en la composición de la flora bacteriana del suelo (Rajaniemia y Allison, 2008). Estos cambios los resienten primero las raíces, por estar en contacto directo con la solución del suelo. Los ápices de las raíces son los primeros en sufrir las consecuencias de los cambios que ocurren en los suelos inundados, por ser los puntos más vulnerables: tienen alta demanda de oxígeno, carecen de paredes celulares secundarias, están expuestos a daños mecánicos en su camino entre las diferentes componentes físicos del suelo (Visser y Pierik, 2007). Swarup et al. (2007) demostraron que el etileno, producido en cantidades elevadas en plantas expuestas a inundación, inhibe el crecimiento de las raíces al influir en la producción de auxinas desde el ápice de las raíces. Por ser los ápices de las raíces los centros de producción de citokininas (Pernisová et al., 2009), su eliminación o daño implica una alteración en el balance hormonal de la planta, que necesariamente debe repercutir en la diferenciación del xilema secundario (Love et al., 2009).

Otros estudios sobre las características fisiológicas de $A$. germinans, han sugerido que poseen una elevada eficiencia en el transporte de agua desde la raíz y principalmente en las hojas (Sobrado, 2000; Sobrado y Ewe, 2006). Considerando lo mencionado anteriormente, es probable que Avicennia germinans también presente variación en la estructura del xilema secundario relacionada con las características ambientales de los sitios en que crece.

La Laguna de La Mancha se localiza en la parte central del estado de Veracruz, México, aproximadamente entre los $19^{\circ} 33^{\prime}$ y $19^{\circ} 36^{\prime} \mathrm{N}$ y los $96^{\circ} 22^{\prime}$ y $96^{\circ} 24^{\prime} \mathrm{O}$, con una extensión de 156 ha (Hernández-Trejo et al., 2006). Durante la época de lluvia hay descargas de agua dulce por medio de arroyos y un aporte permanente por medio de un río que se encuentra en el suroeste del sistema. En las épocas de lluvias y de nortes, la laguna recibe el aporte de agua marina y en período de sequía los pescadores abren la barra; su profundidad varía estacionalmente con un máximo de $1.4 \mathrm{~m}$ cuando se encuentra cerrada la barra y un mínimo de $0.67 \mathrm{~m}$ cuando ésta se abre (Barreiro-Güemes y Balderas-Cortés, 1991). La laguna se encuentra rodeada principalmente por los bosques de mangles, con una extensión de aproximadamente 300 ha (Barreiro-Güemes y Balderas-Cortés, 1991; Hernández-Trejo et al., 2006). En su composición florística sobresalen Avicennia germinans, Rhizophora mangle, Laguncularia racemosa, Conocarpus erecta y Batis maritima, siendo más frecuentes las dos primeras (Hernández-Trejo et al., 2006). Es por esto que se propuso explorar la variación estructural del xilema secundario de A. germinans en la laguna de La Mancha en el estado de Veracruz, México, creciendo en condiciones diferentes de inundación y salinidad y de esta forma identificar los caracteres anatómicos que se relacionan con los factores ambientales. 


\section{Materiales y métodos}

Toma de muestras en campo. Se seleccionaron cinco sitios con diferentes condiciones ambientales en los bosques de mangles que rodean la laguna de La Mancha. Se determinó directamente el nivel de inundación en los sitios con una cinta métrica y el período de inundación con base en Hernández-Trejo et al. (2006). La salinidad de la temporada seca (febrero) y de la temporada húmeda (julio) se determinó tomando muestras de suelo en los diferentes sitios y posteriormente con un medidor de conductividad (Hanna Instruments EC214) en el laboratorio (Cuadro 1).

En cada sitio se tomaron muestras de madera en cuatro árboles que presentaron los diámetros más desarrollados, para lo cual se midió su diámetro a una altura de $1.30 \mathrm{~m}$ sobre el nivel de suelo (Cuadro 1). Las muestras se tomaron utilizando un machete, cortando secciones de albura cercanas al cámbium vascular de aproximadamente $15 \mathrm{~cm}$ de largo por $15 \mathrm{~cm}$ de ancho, a $1.30 \mathrm{~m}$ sobre el nivel del suelo, buscando evitar madera de reacción. Inmediatamente se fijaron en FAA (formaldehído, etanol $96^{\circ}$, ácido glacial acético, agua 10:35:5:50) (Ruzin, 1999).

Proceso de muestras en el laboratorio. Una semana después de recolectar las muestras en el campo y fijarlas en FAA, se enjuagaron con agua corriente y se procedió a colocarlas en GAA (glicerina, etanol $96^{\circ}$, agua 1:1:1) hasta que se seccionaron para obtener cubos de un centímetro cúbico aproximadamente. Posteriormente se obtuvieron cortes transversales, tangenciales y radiales con grosor de 30 a $35 \mu \mathrm{m}$ utilizando un micrótomo de deslizamiento (Yánez-Espinosa et al., 2001). Enseguida se procedió a teñir con safranina y verde fijo siguiendo el método de Johansen (1940) y se montaron con resina sintética para obtener preparaciones permanentes (Ruzin, 1999).

Para la preparación del material disociado se utilizó el método de Jeffrey de maceración de tejidos leñosos (Ruzin,

Cuadro 1. Características de los árboles de Avicennia germinans muestreados (media \pm error estándar) y ambientales de los sitios en La Mancha, Veracruz.

\begin{tabular}{lccccc}
\hline Sitio & Árboles & \multicolumn{3}{c}{ Variables ambientales } \\
\cline { 2 - 6 } & $\begin{array}{c}\text { Diámetro } \\
\text { de tallo } \\
(\mathrm{cm})\end{array}$ & $\begin{array}{c}\text { Período de } \\
\text { inundación } \\
\text { (meses) }\end{array}$ & $\begin{array}{c}\text { Nivel de } \\
\text { inundación } \\
(\mathrm{cm})\end{array}$ & $\begin{array}{c}\text { Salinidad del suelo } \\
(\%)\end{array}$ \\
\cline { 5 - 6 } & & & & $\begin{array}{c}\text { Temporada } \\
\text { seca }\end{array}$ & $\begin{array}{c}\text { Temporada } \\
\text { húmeda }\end{array}$ \\
\hline 1 & $25.65( \pm 4.7)$ & 6 & 160 & 30.09 & 13.80 \\
2 & $32.23( \pm 2.7)$ & 6 & 144 & 19.93 & 8.40 \\
3 & $17.85( \pm 2.3)$ & 3 & 152 & 14.30 & 12.26 \\
4 & $28.10( \pm 2.9)$ & 3 & 62 & 12.57 & 3.71 \\
5 & $15.03( \pm 2.9)$ & 6 & 128 & 9.53 & 3.18 \\
\hline
\end{tabular}

1999). Se elaboraron preparaciones temporales colocando el disociado en glicerina y agua, tiñendo con azul de toluidina.

En esta etapa se realizaron la toma de microfotografías y las mediciones de las muestras. Con este fin, se capturaron imágenes fotográficas digitalizadas con un microscopio compuesto con cámara integrada (Nikon eclipse E600) en los cortes con orientación transversal, radial y tangencial de cada muestra. Posteriormente se realizaron las mediciones con el programa Wincell V5.4., siguiendo las recomendaciones de IAWA (1989). Se realizaron 25 mediciones para longitud, grosor y diámetro de vasos y fibras; y también para ancho y alto de los radios y para obtener el número de vasos y radios, se realizó el conteo en 25 campos respectivamente.

Caracteres anatómicos de la madera. Los caracteres anatómicos de la madera de Avicennia germinans considerados en el análisis fueron: longitud de elemento de vaso $(\mu \mathrm{m})$, diámetro tangencial del lumen vaso $(\mu \mathrm{m})$, grosor tangencial de pared de vaso $(\mu \mathrm{m})$, frecuencia de vasos (número de vasos $\mathrm{mm}^{-2}$ ), proporción de vasos solitarios (\%), proporción de vasos en hileras (\%), diámetro tangencial de punteaduras $(\mu \mathrm{m})$, frecuencia de punteaduras (número de punteaduras $\left.0.25 \mathrm{~mm}^{-2}\right)$, longitud de fibra $(\mu \mathrm{m})$, diámetro tangencial del lumen de fibra $(\mu \mathrm{m})$, grosor tangencial de pared de fibra $(\mu \mathrm{m})$, relación altura de radio $(\mu \mathrm{m}) /$ número de células, relación anchura de radio $(\mu \mathrm{m}) /$ número de células, relación número de radios $\mathrm{mm}^{-1} /$ anchura de radio $(\mu \mathrm{m})$.

Análisis estadístico. El análisis de los datos se llevó a cabo utilizando el análisis discriminante canónico (ADC) que permite identificar las diferencias entre dos o más grupos a partir de medidas de caracteres de los individuos pertenecientes a esos grupos, estimando funciones lineales a partir de las variables cuantificadas, realizando la separación de los grupos de individuos al maximizar la varianza entre los grupos y minimizar la varianza dentro de los grupos. Los coeficientes canónicos estandarizados se utilizan para evaluar la contribución de cada variable independiente en cada función canónica (Pires-da-Silva et al., 2001). También se aplicó un análisis discriminante clasificatorio para verificar los sitios definidos (DISC). Los análisis se llevaron a cabo utilizando el procedimiento PROC CANDISC y PROC DISCRIM respectivamente (SAS Institute, 1989).

Para evaluar las diferencias significativas entre medias de los caracteres anatómicos seleccionados mediante el ADC, se utilizó el modelo II de análisis de varianza (ANVA), de efectos aleatorios evaluados por muestreo de poblaciones normales, y específicamente el modelo II con muestras de clasificación jerárquica o muestreo anidado debido a la estructura de los datos (Snedecor y Cochran, 1970). Esto se puede interpretar como cualquier medida de algún carácter anatómico es igual a la suma de una media general, de una desviación por efecto de sitio, de una desviación por efec- 
to del árbol y de un elemento aleatorio (Yánez-Márquez, 1981). El análisis se llevó a cabo con PROC ANOVA (SAS Institute, 1989). La comparación múltiple de medias estimadas se llevó a cabo a través de la prueba de Tukey (SAS Institute, 1989).

Posteriormente se aplicó un análisis de correlación canónica (ACC) con las variables seleccionadas en la primera función canónica del ADC y las variables ambientales de los sitios, con el propósito de analizar la relación entre dos conjuntos de variables generando dos subconjuntos de variables, uno de variables dependientes (caracteres anatómicos) y otro de variables independientes (variables ambientales), similar a un análisis de regresión múltiple que analiza la relación entre un a única variable criterio y varias variables independientes cuyos valores son conocidos para predecir la variable criterio seleccionada (Hair et al., 1999), pero en la que existen diversas variables en ambos lados de la ecuación. Los conjuntos de variables en cada lado se combinan para producir un valor predictivo que tiene la correlación más alta con el valor predictivo del otro lado (Tabachnick y Fidell, 1989). El análisis se llevó a cabo con PROC CANCORR (SAS Institute, 1989) utilizando los promedios de los árboles en cada sitio y de las variables de los sitios.

\section{Resultados}

En el cuadro 1 se presentan los promedios del diámetro de los árboles muestreados, y los resultados de los análisis de salinidad y las otras variables ambientales de cada sitio consideradas. En estos se puede observar un gradiente de salinidad en donde el sitio 1 presenta la mayor concentración y el sitio 5 la menor.
Cuadro 2. Resumen de los resultados del análisis discriminante canónico de los caracteres anatómicos de Avicennia germinans en La Mancha, Veracruz.

\begin{tabular}{lcccccc}
\hline \multirow{2}{*}{$\begin{array}{l}\text { Función } \\
\text { canónica }\end{array}$} & $\begin{array}{c}\text { Valor } \\
\text { característico }\end{array}$ & \multicolumn{2}{c}{ Proporción } & & \begin{tabular}{c} 
Correlación \\
\cline { 3 - 4 } canónica
\end{tabular} & $p>\mathrm{F}$ \\
\hline Función 1 & 0.541 & 0.42 & 0.42 & 0.35 & 0.0001 \\
Función 2 & 0.453 & 0.36 & 0.78 & 0.31 & 0.0001 \\
\hline
\end{tabular}

El ADC mostró que dos funciones discriminantes explican el 78\% de la varianza total, contribuyendo significativamente a la separación entre sitios (Wilks' $\lambda, \mathrm{F}=10.5, p<0.0001$, $\mathrm{n}=500$ ) (Cuadro 2). Los caracteres anatómicos que contribuyeron significativamente a las dos primeras funciones canónicas se muestran en los coeficientes canónicos estandarizados (Cuadro 3). Estos coeficientes reflejan la contribución conjunta de las variables analizadas para cada función canónica, e individualmente indican la importancia relativa de cada variable (Pires-da-Silva et al., 2001) que fueron diámetro tangencial del lumen de fibra, grosor tangencial de pared de vaso y frecuencia de vasos en la primera función y grosor tangencial de pared de fibra en la segunda función. En la Fig. 1 se pueden observar las medias de clase de las variables canónicas representadas por los números de sitio.

Asimismo, el DISC mostró que los centroides de cada sitio fueron significativamente diferentes $(p<0.0001)$ y el $100 \%$ de las observaciones de los sitios 1, 2, y 5, y el $75 \%$ de los sitios 3 y 4 se clasificaron correctamente.

Los resultados del ANVA revelaron que para los caracteres anatómicos identificados por el ADC, existen diferen-

Cuadro 3. Caracteres anatómicos de Avicennia germinans utilizados en el análisis canónico discriminante y su contribución parcial a las funciones expresados por los coeficientes estandarizados de las funciones discriminantes.

\begin{tabular}{lcc}
\hline Caracteres anatómicos & \multicolumn{2}{c}{ Coeficientes estandarizados } \\
\cline { 2 - 2 } & Función 1 & Función 2 \\
\hline Longitud de elemento de vaso $(\mu \mathrm{m})$ & -0.184 & -0.028 \\
Diámetro tangencial del lumen vaso $(\mu \mathrm{m})$ & -0.077 & -0.095 \\
Grosor tangencial de pared de vaso $(\mu \mathrm{m})$ & $0.468 *$ & 0.235 \\
Frecuencia de vasos $($ número de vasos $\mathrm{mm}-2)$ & $0.603 *$ & 0.192 \\
Proporción de vasos solitarios $(\%)$ & 0.121 & 0.172 \\
Proporción de vasos en hileras $(\%)$ & 0.187 & 0.215 \\
Diámetro tangencial de punteaduras $(\mu \mathrm{m})$ & -0.288 & 0.361 \\
Frecuencia de punteaduras $($ número de punteaduras $0.25 \mathrm{~mm}-2)$ & 0.100 & -0.203 \\
Longitud de fibra $(\mu \mathrm{m})$ & $-0.515 *$ & 0.265 \\
Diámetro tangencial del lumen de fibra $(\mu \mathrm{m})$ & -0.031 & 0.509 \\
Grosor tangencial de pared de fibra $(\mu \mathrm{m})$ & -0.092 & $-0.708 *$ \\
Relación altura de radio $(\mu \mathrm{m}) /$ número de células & 0.242 & -0.121 \\
Relación anchura de radio $(\mu \mathrm{m}) /$ número de células & -0.065 & -0.025 \\
Relación número de radios mm-1/anchura de radio $(\mu \mathrm{m})$ & -0.385 \\
\hline
\end{tabular}

*Caracteres que contribuyen más a la separación de los centroides 


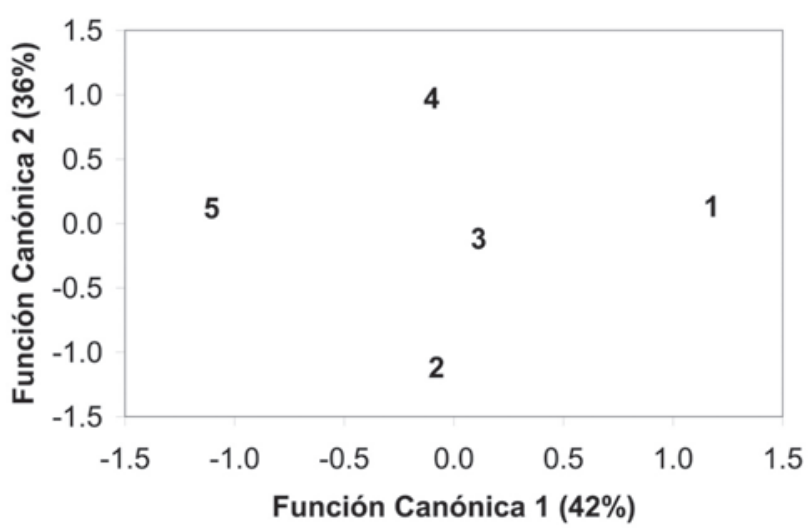

Figura 1. Ordenación de los cinco sitios utilizando los centroides y las dos funciones discriminantes canónicas. cias estadísticas significativas entre las medias de sitios para grosor tangencial de pared de vaso $(\mathrm{F}=13.54, \mathrm{gl}=4, \mathrm{n}=$ $500, p<0.0001)$, frecuencia de vasos $(\mathrm{F}=20.37, \mathrm{gl}=4, \mathrm{n}$ $=500, p<0.0001$ ), diámetro tangencial del lumen de fibra $(\mathrm{F}=35.84, \mathrm{gl}=4, \mathrm{n}=500, p<0.0001), \mathrm{y}$ grosor tangencial de pared de fibra $(\mathrm{F}=26.51, \mathrm{gl}=4, \mathrm{n}=500, p<0.0001)$ (Fig. 2) y estas diferencias entre sitios son las responsables de la mayor parte de la variación total respecto a los árboles muestreados dentro de sitios (Cuadro 4). Solamente para grosor tangencial de pared de vaso y frecuencia de vasos se observaron diferencias entre los sitios 1 y 5 , que son los extremos en el gradiente de mayor a menor salinidad y período de inundación, y también con el grupo de los sitios 2, 3 y 4 que no presentaron diferencias entre ellos, pero si con los otros dos sitios. En la figura 3 se muestran las diferencias en los caracteres anatómicos entre sitios.
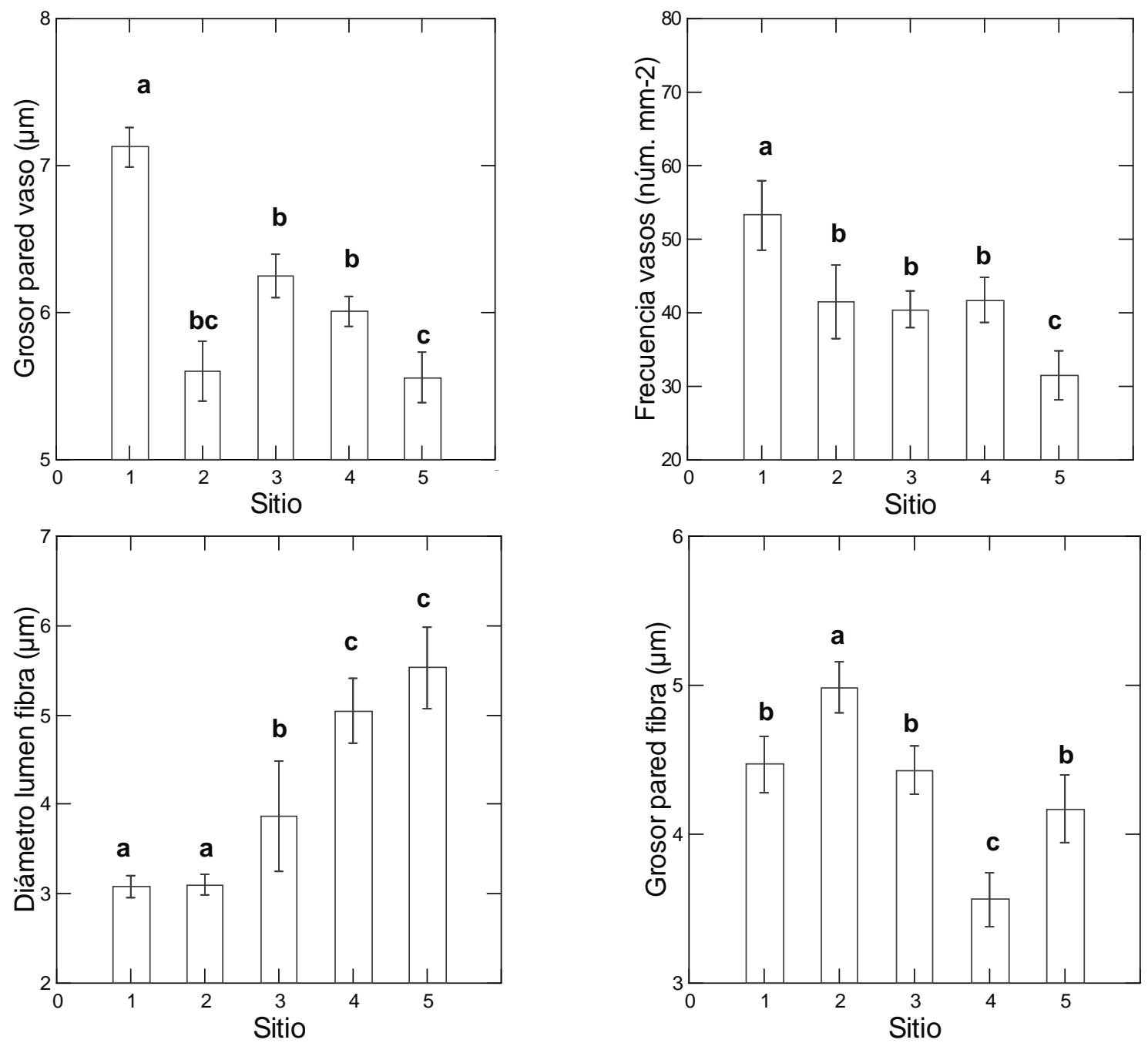

Figura 2. Comparación múltiple de las medias ( \pm error estándar) de los caracteres de la madera de Avicennia germinans seleccionados en el análisis discriminante canónico. letras diferentes representan diferencias significativas (Tukey $p<0.05$ ). 

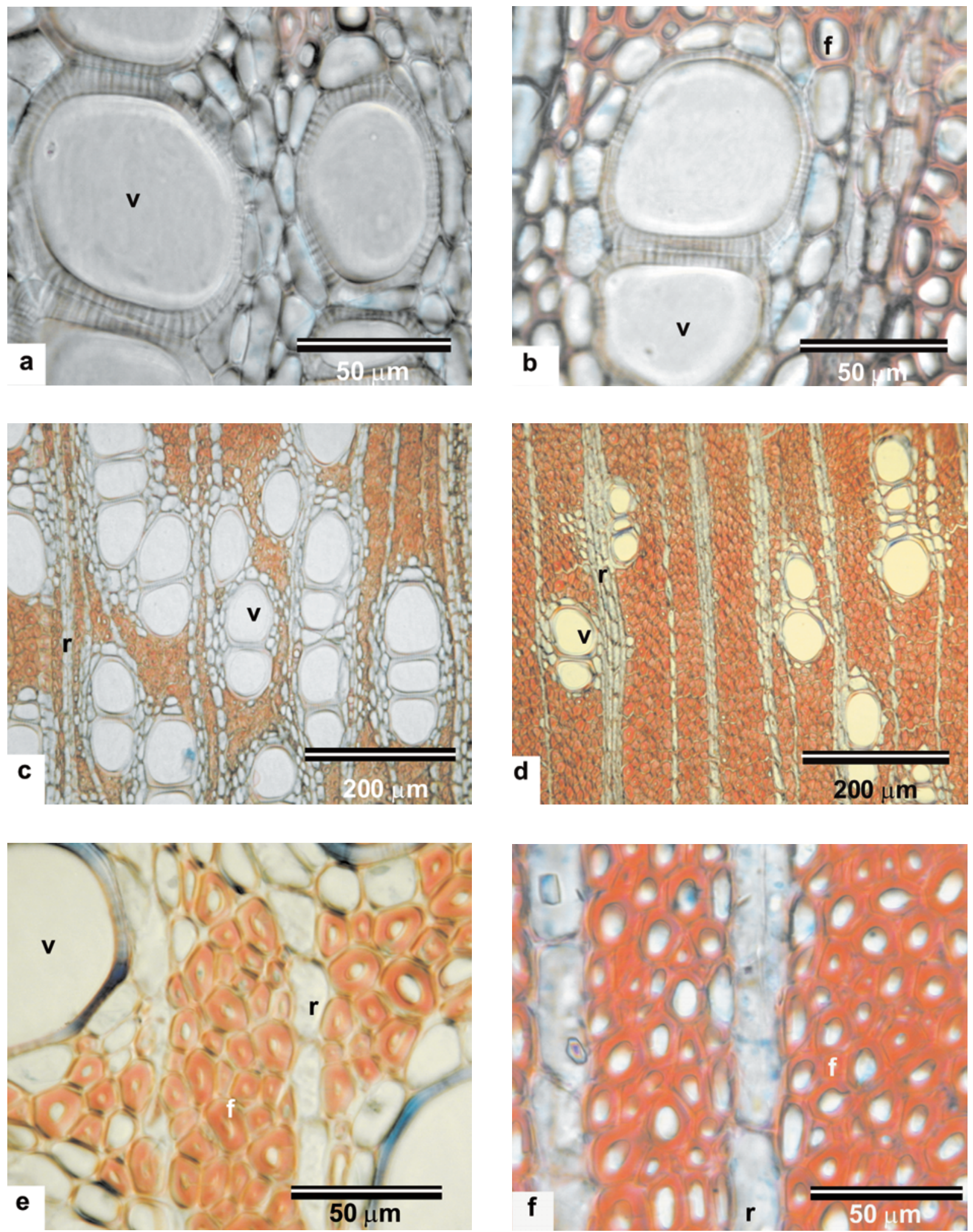

Figura 3. Caracteres anatómicos de la madera de Avicennia germinans seleccionados en el análisis discriminante canónico (Corte transversal). a) Mayor grosor de pared de vaso en sitio uno; b) menor grosor de pared de vaso en sitio cinco; c) mayor frecuencia de vasos en sitio uno; d) menor frecuencia de vasos en sitio cinco; e) menor diámetro de lumen de fibras en sitio uno; f) mayor diámetro de lumen de fibras en sitio cinco. $v=$ vaso; $f=$ fibra; $r=$ radio. 
Cuadro 4. Componentes de la varianza total de los caracteres de la madera de Avicennia germinans seleccionados en el análisis discriminante canónico.

\begin{tabular}{lllll}
\hline Nivel & $\begin{array}{l}\text { Grosor } \\
\text { tangencial } \\
\text { de pared } \\
\text { de vaso }\end{array}$ & $\begin{array}{l}\text { Frecuencia } \\
\text { de vasos }\end{array}$ & $\begin{array}{l}\text { Diámetro } \\
\text { tangencial } \\
\text { del lumen } \\
\text { de fibra }\end{array}$ & $\begin{array}{l}\text { Grosor } \\
\text { tangencial } \\
\text { de pared } \\
\text { de fibra }\end{array}$ \\
\hline Sitio & $\begin{array}{l}1.491 \\
(30.5 \%)\end{array}$ & $\begin{array}{l}236.811 \\
(44.3 \%)\end{array}$ & 5.10 & 0.97 \\
Arbol & 0.408 & 2.499 & 0.55 & $(42.5 \%)$ \\
& $(8.3 \%)$ & $(0.4 \%)$ & $(13.7 \%)$ & $(13.7 \%)$ \\
Determinaciones & $\begin{array}{l}2.996 \\
(61.2 \%)\end{array}$ & $\begin{array}{l}294.97 \\
(55.2 \%)\end{array}$ & $\begin{array}{l}1.00 \\
(43.7 \%)\end{array}$ & 1.00 \\
Total & 4.895 & 534.28 & 2.30 & 2.30 \\
\hline
\end{tabular}

El ACC aplicado a las variables seleccionadas por el ADC y las variables ambientales evaluadas en los sitios muestra que la primera correlación canónica fue de 0.731 (73\% de la varianza, $p<0.008)$ y las correlaciones restantes no fueron significativas $(p<0.1)$. Los datos en el primer par de variables canónicas se pueden observar en el cuadro 5 , mostrando las correlaciones entre las variables originales y las variables canónicas y los coeficientes estandarizados de las variables canónicas. La proporción de la varianza y redundancia indican que el primer par de variables está muy

Cuadro 5. Cargas canónicas cruzadas del primer par de variables canónicas para el conjunto de caracteres anatómicos de la madera de Avicennia germinans y el conjunto de variables ambientales de los sitios de La Mancha, Veracruz.

\begin{tabular}{|c|c|c|}
\hline & \multicolumn{2}{|c|}{$\begin{array}{l}\text { Primer par de variables } \\
\text { canónicas }\end{array}$} \\
\hline & $\begin{array}{l}\text { Correlación } \\
\text { variables } \\
\text { originales y } \\
\text { canónicas }\end{array}$ & $\begin{array}{l}\text { Coeficientes } \\
\text { estandarizados }\end{array}$ \\
\hline \multicolumn{3}{|l|}{ Caracteres anatómicos } \\
\hline Grosor tangencial de pared de vaso & 0.740 & 0.711 \\
\hline Frecuencia de vasos & 0.423 & 0.360 \\
\hline $\begin{array}{l}\text { Diámetro tangencial del lumen } \\
\text { de fibras }\end{array}$ & -0.051 & -0.155 \\
\hline \multicolumn{3}{|l|}{ Variables ambientales } \\
\hline Profundidad & 0.175 & -2.106 \\
\hline Período de inundación & -0.183 & 25.619 \\
\hline Salinidad del agua en período seco & 0.397 & -25.011 \\
\hline $\begin{array}{l}\text { Salinidad del agua en período } \\
\text { húmedo }\end{array}$ & 0.755 & 20.985 \\
\hline Porcentaje de varianza & \multicolumn{2}{|c|}{66} \\
\hline Índice de redundancia & \multicolumn{2}{|c|}{0.887} \\
\hline Correlación canónica & \multicolumn{2}{|c|}{0.731} \\
\hline
\end{tabular}

relacionado. Las cargas cruzadas mostraron que el grosor tangencial de pared de vaso y la frecuencia de vasos están asociados positivamente con la salinidad del agua en la temporada seca y húmeda, y el nivel de inundación y se asocian negativamente con la longitud del período de inundación. Asimismo, el diámetro tangencial del lumen de fibras y el grosor tangencial de pared de fibras se asocian negativamente con la salinidad del agua en la temporada seca y húmeda, y el nivel de inundación y positivamente con la longitud del período de inundación (Cuadro 5).

\section{Discusión}

La relación negativa entre frecuencia de vasos y diámetro de vaso se presenta en Avicennia germinans, lo que implica una compensación entre eficiencia hidráulica y estabilidad mecánica del xilema, como se ha observado en otras especies de mangle (Yáñez-Espinosa et al., 2001, 2004; Schmitz et al., 2006). Asimismo, se ha probado que la estructura del sistema vascular del tallo en varias especies de árboles, tiene una fuerte influencia en la estructura y funcionamiento de las hojas, la ganancia de carbono, la eficiencia en el uso de nutrientes y la tasa decrecimiento (Zahng y Cao, 2009). Los vasos numerosos favorecen la protección contra la cavitación en ambientes estresantes, particularmente asociado al incremento en salinidad y disponibilidad de agua (Schmitz et al., 2006), como ocurre con A. germinans que crece en sitios con mayor período de inundación y salinidad en el período húmedo, y menor nivel de inundación y salinidad en la temporada seca. En un estudio realizado en Avicennia marina, la mayor frecuencia de vasos no solo incrementa la eficiencia hidráulica, sino también salvaguarda el sistema de transporte de agua comparado con una red con menor frecuencia de vasos, con una mayor proporción de vasos permaneciendo funcionales para el mismo número de vasos embolizados (Robert et al., 2009). En otro estudio con Laguncularia racemosa se observó también la relación de mayor frecuencia de vasos en sitios con salinidad elevada y mayor nivel de inundación, y se sugiere que el daño por cavitación en el xilema podría presentarse cuando el nivel de inundación es elevado, aumentando la posibilidad de que ocurra una sequía esporádica (Yáñez-Espinosa et al., 2004). La conductancia hidráulica especifica tallo-hoja es bajo en A. germinans y el uso conservador del agua asociado con una baja eficiencia de transporte puede tener ventajas en las zonas con mayor salinidad, donde la regulación de la perdida de agua puede tener considerable influencia en la cantidad de sal que concentra la hoja (Sobrado, 2000).

Particularmente Avicennia germinans es capaz de absorber agua en suelos hipersalinos a través del establecimiento de gradientes de potencial hídrico amplios entre el suelo y las hojas (Waisel, 1972; Suárez et al., 1998; Sobrado y Ewe, 2006), por lo que el aumento del grosor de la pared de los vasos en sitios con mayor salinidad en la temporada húmeda 
y el período de inundación sugiere que es significativamente funcional para ofrecer suficiente fuerza mecánica para resistir presiones negativas fuertes (Baas et al., 1983).

El incremento del diámetro de lumen de fibra de Avicennia germnans se asocia con sitios con menor salinidad en la temporada húmeda, y se observa la tendencia inversa en el grosor de la pared de fibra. De acuerdo con Sun y Suzuki (2001) las dimensiones de las fibras tienen una influencia considerable en el soporte mecánico de los tallos, lo que implica que el incremento en el grosor de la pared de la fibra y la disminución en el diámetro de lumen, aumenta la resistencia mecánica. Esto sugiere que los árboles que crecen en sitios con mayor salinidad en el período húmedo presentarían menor resistencia mecánica, lo que ya se había sugerido para Laguncularia racemosa creciendo en las mismas condiciones y que les permitiría resistir la inestabilidad de los suelos con inundación prolongada (Yáñez-Espinosa et al., 2004). Jacobsen et al. (2005) mencionan que existe correlación positiva entre resistencia a la cavitación de los vasos y grosor de la pared de las fibras de xilema, pero negativa con el área de lumen de la fibra. Particularmente, las fibras pueden atenuar el riesgo de implosión de los vasos, reduciendo el estrés de la deflexión de la membrana de la punteadura o el estrés que conduce al colapso de los vasos y la microfractura de la pared celular.

En diversos estudios de Avicennia germinans se menciona la existencia de variación en caracteres morfológicos y fisiológicos de los árboles y caracteres morfológicos en las hojas que corresponderían al estrés hídrico de larga duración asociado al aumento de salinidad en el sitio (Soto y Corrales, 1987; Soto, 1988; Blanco y Flores, 2003; Suárez y Medina, 2005). En estos se detectaron la reducción en la altura total de las plantas como respuesta a la salinidad elevada, así como la reducción en el área foliar en condiciones ambientales donde es difícil la obtención de agua del suelo (Soto y Corrales, 1987). También se ha detectado la conductancia hidráulica especifica tallo-hoja en estas condiciones de salinidad elevada (Sobrado, 2000), lo que se explica por el aumento en la frecuencia de vasos y su disminución en diámetro en el xilema del tallo, y al mismo tiempo el mayor grosor de pared de vaso implica que resisten presiones negativas fuertes.

En estudios anteriores no se detectaron diferencias estadísticamente significativas entre sitios para los caracteres anatómicos de frecuencia de vasos, grosor de pared de vaso, diámetro de lumen de fibra y grosor de pared de fibra, presentes en un intervalo de salinidad entre 6 y $10 \%$ (YáñezEspinosa et al., 2001). Sin embargo, los resultados de este estudio muestran diferencias estadísticamente significativas entre estos caracteres presentes en sitios con salinidad de 3 y $30 \%$, pero no existen diferencias en los sitios con un intervalo de salinidad entre 10 y $20 \%$. Esto sugiere que si debido al calentamiento global el nivel del mar se eleva e invade los sitios en los que se encuentra creciendo A. germi- nans, la salinidad podría afectar su eficiencia en la conducción hidráulica ocasionando disminución en su crecimiento, pero le permitiría mantener una distribución más amplia que otras especies de mangle.

\section{Agradecimientos}

La presente investigación fue financiada con fondos del Instituto de Ecología, A.C., durante el desarrollo del posdoctorado de la autora. Agradecemos a Víctor Vázquez por su ayuda con el análisis de salinidad.

\section{Literatura citada}

Baas P. 1982. Systematic, phylogenetic and ecological wood anatomy: history and perspectives. En: Baas P. Ed. New perspectives in wood anatomy, pp.23-58, Nijhoff, The Hague.

Baas P. 1986. Ecological patterns in xylem anatomy. En: Givnish T.J. Ed. On the economy of plant form and function, pp.327352, Cambridge University Press, Cambridge.

Baas P., Werker E. y Fahn A.1983. Some Ecological Trends in vessel characters. IAWA Bulletin 4:141-147.

Barreiro-Güemes T. y Balderas-Cortés J. 1991. Evaluación de algunas comunidades de productores primarios de la Laguna de la Mancha, Veracruz. Anales del Instituto de Ciencias del Mar y Limnología. Universidad Nacional Autónoma de México, 18:229-245.

Blanco, J.F. y Flores, L.A. 2003. El tamaño y la forma si importan: variación del área foliar de dos especies de mangle en relación al gradiente de salinidad en la Laguna La Mancha, Veracruz. Curso de Ecología de ecosistemas costeros. Organization for Tropical Studies, Louisiana State University e Instituto de Ecología, A.C pp.92-96

Carlquist S. 1988. Comparative wood anatomy. Springer, Berlin, Heidelberg, New York

Chen H., Qualls R.G. y Blank R.R. 2005. Effect of soil flooding on photosynthesis, carbohydrate partitioning and nutrient uptake in the invasive exotic Lepidium latifolium. Aquatic Botany 82:250-268.

Field C.D. 1995. Impact of expected climate-change on mangroves. Hydrobiologia 295:75-81.

Hair J.F., Anderson R.E., Tatham R.L. y Black W.C. 1999. Análisis multivariante. Prentice Hall Iberia. Madrid.

Hernández-Trejo H., Priego-Santander A.G., López-Portillo J.A. e Isunza-Vera E. 2006. Los paisajes físico-geográficos de los manglares de la laguna de La Mancha, Veracruz, México. Interciencia 31:211-219.

IAWA [International Association of Wood Anatomists] Committee 1989. IAWA list of microscopio features for hardwood identification. IAWA Bulletin n.s. 10: 219-332.

Jacobsen A.L., Ewers F.W., Pratt R.B., Paddock III W.A. y Davis S.D. 2005. Do xylem fibers affect vessel cavitation resistance? Plant Physiology 139:546-556.

Jiménez J.A. y Lugo A.E. s.f. Avicennia germinans (L.) L. SOITF-SM-4. Department of Agriculture, Forest Service, Southern Forest Experiment Station. New Orleans, LA, U.S.

Johansen D.A. 1940. Plant microtechnique. McGraw-Hill, New York.

Lacerda L.D., Conde J.E., Bacon P.R., Alarcon C., Alvarez-Leon 
R., D’Croz L.D., Kjerfve B., Polania J. y Vannucci M. 1993. Ecosistemas de Manglar de América Latina y el Caribe. En: Lacerda D. Ed. Conservación y Aprovechamiento Sostenible de Bosques de Manglar en las Regiones de América Latina y Africa, pp.1-39, International Tropical Timber Organization y International Society for mangrove Ecosystems, Okinawa Japón.

López-Portillo J. y Ezcurra E. 2002. Los manglares de México: una revisión. Madera y bosques Número especial:27-51.

Love J., Björklund S., Vahala J., Hertzberg M., Kangasjärvi J.y Sundberg B. 2009. Ethylene is an endogenous stimulator of cell division in the cambial meristem of Populus. PNAS 106:59845989.

Pernisová M., Klíma P., Horák J., Válková M., Malbeck J., Souček P., Reichman P., Hoyerová K., Dubová J., Friml J., Za;žímalová E. y Hejátko J. 2009. Cytokinins modulate auxin-induced organogenesis in plants via regulation of the auxin efflux. PNAS 106:3609-3614.

Pires-da-Silva A., Imhoff S., Giarola N.F.B. y Tormena C. 2001. Análisis multivariado y univariado en la discriminación de sistemas de uso de suelos del centro de Santa Fe. Edafología 8: 21-34.

RajaniemiaT.K. y Allison. V.J. 2008. Abiotic conditions and plant cover differentially affect microbial biomass and community composition on dune gradients. Soil Biology and Biochemistry 41:102-109.

Robert E.M.R., Koedam N., Beeckman H. y Schmitz N. 2009. A safe hydraulic architecture as wood anatomical explanation for the difference in distribution of the mangroves Avicennia and Rhizophora. Functional Ecology 23:649-657.

Ruzin S.E. 1999 Plant microtechnique and microscopy. Oxford University Press. New York.

SAS Institute. 1989. SAS Procedures Guide, Ver. 6.04. SAS Institute. North Carolina.

Schmitz N., Verheyden, A., Beeckman, H., Gitundu Kairo, J. y Koedam, N. 2006. Influence of a salinity gradient on the vessel characters of the mangrove species Rhizophora mucronata. Annals of Botany 98: 1321 - 1330.

Snedecor G.W. y Cochran W.G. 1970. Métodos estadísticos. CECSA. México, D.F.

Sobrado M.A. 2000. Relation of water transport to leaf gas exchange properties in three mangrove species. Trees 14:258-262.

Sobrado M.A. 2001. Hydraulic properties of a mangrove Avicennia germinans as affected by $\mathrm{NaCl}$. Biologia Plantarum 44:435-438.

Sobrado M.A. y Ewe S.M.L. 2006. Ecophysiological characteristics of Avicennia germinans and Laguncularia racemosa coexisting in a scrub mangrove forest at the Indian River Lagoon, Florida. Trees 20:679-687.

Soto R. 1988. Geometry, biomass allocation and leaf life-span of Avicennia germinans (L.) L. (Avicenniaceae) along a salinity gradient in Salinas, Puntarenas, Costa Rica. Revista. Biología
Tropical 36:309-324.

Soto R. y Corrales L.F. 1987. Variación de algunas características foliares de Avicennia germinans (L.) L. (Avicenniaceae) en un gradiente climático y de salinidad. Revista. Biología Tropical 35: 245-256.

Suárez N. y Medina E. 2005. Salinity effect on plant growth and leaf demography of the mangrove Avicennia germinans L. Trees 19:722-728.

Suárez N., Sobrado M.A y Medina E. 1998. Salinity effects on the leaf water relations components and ion accumulation patterns in Avicennia germinans (L.) L. seedlings. Oecologia 114:299304.

Sun Q. y Suzuki M. 2001. Quantitative character variation of cambial derivatives in mangroves and their functional significance. Trees 15:249-261.

Swarup R., Perry P., Hagenbeek D., Van Der Straeten D., Beemster G.T.S., Sandberg G., Bhalerao R., Ljung K. y Bennett M.J. 2007. Ethylene upregulates auxin biosynthesis in Arabidopsis seedlings to enhance inhibition of root cell elongation Plant Cell 19:2186-2196.

Tabachnick B.G. y Fidell L.S. 1989. Using multivariate statistics. Harper Collins. New York.

Tomlinson P.B. 1986. The botany of mangroves. Cambridge University Press. New York.

Visser E.J.W. y Pierik R. 2007. Inhibition of root elongation by ethylene in wetland and non-wetland plant species and the impact of longitudinal ventilation. Plant, Cell and Environment 30:31-38.

Von Prahl H., Cantera, J. y Contreras, R. 1990. Manglares y hombres del Pacífico Colombiano. Editorial Presencia, Colombia.

Waisel, Y. 1972. Biology of halophytes. Academic Press. New York.

Yáñez-Espinosa L., Terrazas T. y López-Mata L. 2001. Effect of flooding on wood and bark anatomy of four species in a mangrove forest community. Trees 15:91-97.

Yáñez-Espinosa L., Terrazas T., López- Mata L. y Valdez-Hernández, J.I. 2004 Wood variation in Laguncularia racemosa and its effect on fibre quality. Wood Science and Technology 38:217226.

Yánez-Márquez O. 1981. Estudio de la variación de algunas características de Pinus strobus var. chiapensis Martínez de tres localidades de su distribución natural. Tesis profesional. Departamento de Bosques, Universidad Autónoma Chapingo, México, $173 \mathrm{pp}$.

Zhang S., Baas P. y Zandee M. 1992. Wood structure of the Rosaceae in relation to ecology, habit and phenology. IAWA Bulletin 13:307-349

Zhang J.L. y Cao K.F. 2009. Stem hydraulics mediates leaf water status, carbon gain, nutrient use efficiencies and plant growth rates across dipterocarp species. Functional ecology 23:658-667 